\title{
La enseñanza del juicio moral en Bachillerato con la película El chacal de Nahueltoro (1969)
}

Teaching moral judgement in upper-middle school based on the movie The jackal of Nahueltoro (1969)

Juan Manuel Arriaga Benítez ${ }^{l}$

\begin{abstract}
RESUMEN
El juicio moral es un tema abordado en la asignatura de Ética, que se imparte en las escuelas mexicanas de nivel Medio Superior (Bachillerato, Preparatoria o equivalentes), para que los estudiantes adquieran conocimientos sobre formación de valores y actitudes, pero sobre todo para que obtengan una comprensión más detallada del proceso cognitivo que acompaña la configuración de una moral congruente con su edad y calidad de vida. El objetivo de la presente investigación es demostrar cómo la película chilena El chacal de Nahueltoro induce un aprendizaje más significativo de este tema matriz en la formación ética de los estudiantes de entre 15 y 17 años de edad. Mi necesidad de escribir este artículo surgió como un interés por fomentar en la comunidad global docente la revisión e inclusión de materiales fílmicos dentro del aula de clases, ya que he notado que el aprendizaje a través del cine es un incentivo adecuado para captar el interés de los estudiantes en un determinado tema; para ello, se describen y evalúan, mediante un enfoque cualitativo de tendencia funcionalista, los resultados didácticos que he tenido oportunidad de obtener a lo largo de mis dos últimos años como docente, al facilitar la enseñanza mediante la discusión y el análisis de la mencionada película en el ambiente de clase. La línea científica que sigo está basada en el proceso de investigación cualitativa explicado por Roberto H. Sampieri en su Metodología de la investigación.
\end{abstract}

Palabras clave: Aprendizaje; didácticas; cine; enseñanza; ética.

\section{ABSTRACT}

Moral judgement is a topic studied in Ethics discipline, which belongs to High School (Preparatory or equivalents) in Mexico and whose purpose is that students acquire the knowledge about the formation of values and attitudes, but especially that they get a more detailed understanding of the cognitive process that follows the configuration of a moral consistent with their age and quality of life. The purpose of this investigation is to demonstrate how the Chilean film The jackal of Nahueltoro induces a more significant knowledge about this leading topic in the ethic formation of students between 15 and 17 years old. My necessity of writing this paper arose as an interest to promote in the global teaching community the review and insertion of filmic material within the classroom, because I have noticed that learning through film becomes an appropriate incentive for attracting the interest of students in a certain subject. To meet this goal, I describe and evaluate with a functionalist tendency qualitative approach the didactic guidelines that I have been able to obtain throughout my last two teaching years by making easier tuition considering discussion and analysis of the aforementioned film in the class environment. The scientific line that I follow is based on the process of qualitative investigation explained by Roberto H. Sampieri in his Methodology of investigation.

Keywords: Learning; didactics; films; teaching; ethics.

\footnotetext{
${ }^{1}$ Profesor en el Colegio de Estudios Científicos y Tecnológicos; doctorando en el programa de Doctorado en Letras de la Universidad Nacional Autónoma de México; miembro fundador de la OIR (Organización Iberoamericana de Retórica); primer lugar del "Certamen Internacional de Ensayo" de la COCOPA (Comisión Bicameral de la Concordia y la Pacificación) en la categoría de Ensayo Político. Correo electrónico: hector_aquiles_apolo@ hotmail.com
} 


\section{Planteamiento, hipótesis metodología}

La opera prima del cineasta chileno Miguel Littín tuvo un enorme éxito en taquilla. Brutal y directa, esta producción fílmica, basada en hechos reales acontecidos en la locación de Nahueltoro, Chile, presenta una estructura y una construcción de personaje que se amoldan a la perfección para la enseñanza del Juicio Moral en la asignatura de Ética que se imparte en el Bachillerato.

La película posee afinidad con el cine documental, no sólo debido a que su rodaje se llevó a cabo en locaciones donde ocurrieron los asesinatos perpetuados por Jorge Valenzuela Torres, quien a la postre sería apodado como El chacal por la crudeza con que los medios de comunicación determinaron la naturaleza de los acontecimientos, sino también debido a la naturalidad con que la cámara filma la recreación de este evento histórico que, al parecer, se ha consolidado como un hito delictivo en la historia de Chile y cuya estructura es capaz de admitir variadas interpretaciones.

En las clases de Ética, esta película ha permitido centrar la atención de mis estudiantes en el dilema ético que plantea: ¿Qué condiciones determinan que una persona sea "malvada"? ¿Las leyes perciben al crimen de manera absoluta para todas criminales? ¿Al nivel de la realidad, quiénes pueden considerarse realmente buenos y quiénes malos?

Las respuestas a estas preguntas me llevaron a la siguiente hipótesis con la que di comienzo a mis primeras observaciones: un análisis basado en estudio de caso resaltará una interpretación congruente de cada estudiante, aunque cada uno tenga una lectura diferente de él; coincidirán en una aplicación correcta de su conocimiento sobre juicio moral tras retomar los fundamentos establecidos con base en los estudios de Jean Piaget (1987) y Lawrence Kohlberg (1992) en el ámbito de la psicología moral; en consecuencia, la película les proporcionará herramientas para romper el prejuicio de que el bien y el mal son nociones morales absolutas (siendo éste el objetivo de la secuencia didáctica). La muestra estudiantil fue de 36 estudiantes sobre una población de 3 grupos de exactamente 30 alumnos cada uno.

Sin embargo, una vez que se comenzaron a obtener los primeros datos sobre la interpretación del filme, fue necesario dimensionar nuevamente el marco teórico y la metodología de enseñanza sobre juicio moral previa al visionado de la película, de modo que pudiera, para desarrollar mejor el tema en posteriores semestres, operar un contenido más eficiente en lecciones respectivas; surgió así un modelo teórico de mi autoría, cimentado en los estudios del espectro psicológico del desarrollo moral a través de los estadios o etapas que lo configuran mediante el uso de recursos comparativos y descriptores de comportamiento que tienen la siguiente configuración lectiva para los cuatro primeros estadios y que se proporcionan al alumno como un archivo impreso al que se le adaptan observaciones propias: 


\begin{tabular}{|c|c|}
\hline $\begin{array}{l}\text { ESTADÍO } 1 \\
\text { Absolutismo y } \\
\text { orientación al } \\
\text { castigo }\end{array}$ & $\begin{array}{l}\text { - Ingenuo realismo moral } \\
\text { Considera el significado de una situación como una cualidad inherente física- } \\
\text { mente a la acción. } \\
\text { - Aplicación literal de etiquetas o reglas } \\
\text { Es la tendencia a tomar de forma absoluta las acciones sin tener en cuenta } \\
\text { circunstancias mediadoras que alteren su significado moral. } \\
\text { - Marcada orientación al castigo } \\
\text { - Incapacidad para distinguir perspectivas sociales } \\
\text { Se refiere a la incapacidad para tomar en cuenta otros puntos de vista. } \\
\text { - Unilateralidad } \\
\text { La justicia se basa en relaciones de poder o status. }\end{array}$ \\
\hline $\begin{array}{c}\text { ESTADÍO } 2 \\
\text { Individualismo } \\
\text { instrumental }\end{array}$ & $\begin{array}{l}\text { - Se descubre que existen conflictos de intereses } \\
\text { Las acciones morales exigen reciprocidad, es decir, el individuo se da cuenta } \\
\text { que el absolutismo no es una forma de justicia adecuada e intenta establecer } \\
\text { soluciones a partir de intercambios materiales. } \\
\text { - Satisfacción de necesidades personales } \\
\text { El individuo se preocupa por proteger sus propios intereses, intentando reducir } \\
\text { las consecuencias negativas de sus actos y satisfacer las necesidades de la } \\
\text { forma más fácil posible. }\end{array}$ \\
\hline $\begin{array}{c}\text { ESTADÍO } 3 \\
\text { La moral de la } \\
\text { normativa inter- } \\
\text { personal }\end{array}$ & $\begin{array}{l}\text { - Adopción de la perspectiva de una tercera persona } \\
\text { Se supera el individualismo y los intereses de satisfacción personal al saber } \\
\text { que otros individuos tienen otros puntos de vista. } \\
\text { - Expectativas morales compartidas } \\
\text { Se establecen relaciones de confianza mutua y no a nivel necesariamente } \\
\text { material. } \\
\text { - Orientación a las relaciones interpersonales } \\
\text { Los individuos se preocupan por pertenecer a grupos, como el noviazgo o un } \\
\text { equipo, según perspectivas convencionales comunes (estereotipos y gustos). } \\
\text { - Mayor nivel de reciprocidad } \\
\text { La justicia se basa en la empatía ("No hagas a otros lo que no quieres que te } \\
\text { hagan a ti"). }\end{array}$ \\
\hline $\begin{array}{c}\text { ESTADÍO } 4 \\
\text { La moral de la } \\
\text { coherencia y el } \\
\text { sistema social }\end{array}$ & $\begin{array}{l}\text { • La sociedad como un todo } \\
\text { Los intereses individuales se vuelven congruentes con los sistemas sociales y } \\
\text { son válidos sólo si se toma en cuenta el bien común. } \\
\text { • Preocupación por la coherencia y la imparcialidad } \\
\text { Se desarrolla el respeto por los demás y la participación activa e imparcial en } \\
\text { la solución de conflictos de intereses. } \\
\text { - Justicia basada en deberes y derechos } \\
\text { Se busca cumplir con las obligaciones impuestas por las leyes, actuando con } \\
\text { base en las reglas o normas impuestas por la sociedad. El individuo se ve a sí } \\
\text { mismo como un miembro de la sociedad. }\end{array}$ \\
\hline
\end{tabular}

No es mi intención, sin embargo, profundizar en el contenido que reciben los estudiantes sobre el tema, de modo que en este artículo me ceñiré a describir los resultados que me permiten evaluar cualitativamente cómo la película chilena El chacal de Nahueltoro induce un aprendizaje más significativo de ese tema matriz en la formación ética de los estudiantes de Bachillerato; sin embargo, es de resaltar que las fuentes referenciadas psico- 
logía moral son precisamente el núcleo que constituye la información que utilizo para la enseñanza del juicio moral.

Mi hipótesis, con base en mi segundo muestreo (el que constituye el segundo año de enseñanza de la asignatura de Ética), se modificó a la luz de los primeros resultados, quedando establecida de la siguiente manera: el aprendizaje sobre juicio moral se denota mediante el empleo de material fílmico basado en una metodología de estudio de caso (la cinta chilena $E l$ chacal de Nahueltoro), de modo que los estudiantes aplicarán sus conocimientos previos sobre el tema en la discusión sobre los factores que influyen en la transición de un estadio a otro y en la ruptura de los prejuicios sobre las nociones de bien y mal. Sobre esta hipótesis y el respectivo muestreo estudiantil, que constituye 30 alumnos de una población de dos grupos con 39 y 38 estudiantes, se describen los resultados en las subsiguientes partes de este artículo.

Para la elección del material fílmico que se utiliza como estudio de caso para este tema, se tomaron en cuenta los siguientes criterios: la película debía mostrar un dilema moral que resaltara la controversia entre la culpabilidad y la inocencia de un personaje, o bien, el grado de maldad con que se pudiera juzgar una acción, dadas las convenciones con que se define un crimen y los prejuicios en torno a condicionantes sociales (SÁNCHEZ, 1999, p. 162); igualmente, el material a proyectar debía inducir una respuesta emocional basada en perspectivas diferentes y vigencia de valores (MIRAVALLES, 1998, p. 63), como la pro- porcionada por algún otro personaje, un medio de comunicación o una narrativa en off, de modo que las opiniones se polarizaran, pero sin el afán de resultar contradictorias; por último, dicho material tendría que mostrar al personaje central mediante un arco argumental que distinguiera las etapas del juicio moral de forma parcialmente evidente, de modo que los estudiantes enfocaran sus discusiones en las causas, de modo que las consecuencias de los actos de tal personaje satisficieran sus aproximaciones al tema previamente abordado. La estética narrativa de El chacal de Nahueltoro y su estructura, analizadas desde un paradigma funcionalista, ofrecían la posibilidad de mantener la atención a la narrativa y a la vez el estilo de dramatización de los eventos históricos satisfacían mi interés por "enganchar" a los estudiantes mediante la empatía. A este respecto, el esquema que plantea TOBÍAS, DUARTE Y KEMCZINSKI resultó revelador y se adaptó muy bien a mis criterios de enseñanza (2015, p. 66).

El material bibliográfico con que cuento para la realización de la presente investigación tiene la característica central de proporcionar información sobre por qué la enseñanza de esta asignatura se enriquece a partir de la inclusión de una película en el aula de clases, de modo que se denote tanto la labor del docente como la del alumno en la consecución de aprendizajes significativos. Los trabajos citados dentro del cuerpo del artículo harán relevante el tema matriz, juicio moral, y me permitirán a la vez fundamentar un modelo para su enseñan- 
za, al sobrepasar los límites que establece la sola intención ejemplificativa para posicionar el interés de los alumnos en un nivel de reflexión crítica en el ámbito del estudio de caso.

En el aspecto metodológico, me sirvo de los fundamentos de investigación cualitativa expuestos por Roberto H. Sampieri (2018), por considerarla sencilla, asequible y actualizada, además de ser una herramienta de investigación inteligible para estudiantes de Bachillerato y útil en la docencia.

\section{El juicio moral: una herramienta base en el entendimiento de la Ética y el autoconocimiento}

Una adecuada enseñanza del juicio moral en el Bachillerato estriba en el modo en que se le conceptualice sin que el profesor entre en conflicto con sus propios prejuicios. En el nivel Medio Superior de educación mexicana, según me ha parecido a partir de mi experiencia docente de los últimos dos semestres en que he impartido la asignatura de Ética, el juicio moral es no sólo un fundamento teórico con que el alumno revisa su sistema de valores, sino también es una herramienta para juzgar las implicaciones sociales y emocionales que en el resto del currículo de Ética se estudian y, por extensión, también en el de las asignaturas correspondientes a las Humanidades y las Ciencias Sociales. Un claro ejemplo de ello es el enfoque con que se abordan las implicaciones de la madurez sexual dentro del espacio de interacción social; el abordaje implica hacer una distinción entre la madurez biológica y la madurez emocional so- bre el referente de la sexualidad, por lo cual es imprescindible que un alumno fundamente las observaciones que infiere sobre sexualidad en su conocimiento acerca de los estadios del juicio moral.

Jean Piaget fundamentó su estudio sobre los estadios morales del niño con base en las relaciones que éstos tienen con su contexto (PÉREZ et al., 1996, p. 137); explica las circunstancias que rodean el paso del segundo al tercer estadio del juicio moral como un vínculo que establece relaciones de acuerdo mutuo (1984, p. 33), es decir, los niños (de entre 7 y 10 años) tienden a conducir su pensamiento moral a la consideración de la perspectiva de una tercera persona; este aspecto del tercer estadio es crucial para entender el cuarto estadio, que es precisamente aquél en el que la muestra de mi población estudiantil se encuentra cuando ingresa al semestre en que se cursa Ética, por lo que su juicio del entorno básicamente queda delimitado a considerar no sólo las perspectivas de los demás y reaccionar emocionalmente a los estímulos (estadio 3), sino también a considerar que "las reglas del juego", en palabras de Piaget (ibid., p. 38), consideran las fronteras sociales en las que su interés va más allá de la cooperación para posicionar el razonamiento moral sobre la regla en sí (ibid., p. 40). En otras palabras, mi muestra de alumnos, al estudiar el tema del juicio moral, ha alcanzado un estado de consciencia del estadio moral correspondiente a su edad (estadio 4) mediante una revisión de sus factores determinantes. 
Este autoconocimiento, explicado de tal modo que transmisión de la información supere los aspectos descriptivos, les permite juzgar de forma más clara sucesos y razonamientos que pertenecen a estadios del juicio moral que preceden a éste. Por ejemplo, los resultados me revelaron que, si un estudiante ha comprendido que a sus 15 o 16 años está capacitado moralmente para juzgar a las instituciones sociales de su sistema político, le será perspicuo entender por qué los niños de 4 o 5 años, pertenecientes a un estadio inferior, no cuestionan racionalmente las autoridades de quienes les imponen reglas o castigos, o por qué no existe una responsabilidad en el hecho moral, según Piaget (VILLEGAS, 1998, p. 224). Del mismo modo, he podido inferir que los alumnos con mejor autoestima son capaces de relacionar de forma más explícita el valor emocional de un objeto que les fue obsequiado, debido a que el estadio 3, en el que se rastrean las relaciones interpersonales, constituyó para ellos una etapa que los volvió moralmente aptos para estimar adecuadamente las convenciones de amistad o noviazgo.

Con base en estas inferencias, ver y analizar la película El chacal de Nahueltoro tuvo una incidencia educativa bastante satisfactoria entre los alumnos de la segunda población, pues ilustró la influencia de los conflictos (GRUP EMBOLIC, 1998, p. 78) que suscitan las divergencias morales entre individuos y ejemplificó cómo las circunstancias y los contextos son los factores de evolución moral que permiten el tránsito de un estadio a otro.
La película, de hecho, propone primeramente un acercamiento al subtexto narrativo de manera externa al conflicto en el que se encuentra el personaje principal, Jorge Valenzuela, pero que conduce al espectador ex professo por un camino de falsa objetividad: la primera escena muestra una cobertura mediática sobre el asesinato de una familia conformada por una viuda y sus 5 hijos; se nos permite saber que el asesino es el señor Valenzuela a quien los medios de comunicación denotan de asesino, acaparando todo el escenario visual en un intento por obtener respuestas suyas que arrojen luz sobre la brutalidad de los acontecimientos. La población estudiantil, durante este desenvolvimiento mediático, responde emocionalmente con indignación y desprecio hacia el perpetuador del crimen, simpatizando con la opinión pública que imponen los medios informativos, ofreciendo así una visión del estereotipo criminal que, en lo posterior, acentuará paradójicamente el aspecto crítico al que alude Del Moral (1997, p. 150), al tener ya el alumno un mejor referente sobre los factores determinantes de su condición mora: la infancia y juventud del personaje central.

La película, por ende, avanzar hasta un punto en el que los alumnos comprenden que el asesino es realmente un campesino ignorante y analfabeta al que las circunstancias de su entorno no le permitieron acceder al desarrollo moral característico de los estadios superiores al segundo, sobre todo al notar ellos la falta de congruencia entre sus acciones o su lenguaje y su edad, por lo que comprenden así la 
incapacidad del personaje para sentir empatía o, incluso, entender lo que significa un crimen.

La película capta la atención del estudiante desde la primera escena, pues despierta la curiosidad del alumno mediante una apertura emocional esperada (GRUP EMBOLIC. 1998, p. 78); es decir, la película logra hacer sentir aquello que pretende $\mathrm{y}$ abre un panorama de expectativas que, según he observado en las interpretaciones de los estudiantes muestra, inducen a una posterior reconsideración de los propios juicios iniciales $\mathrm{y}$, por ende, fomentan asociaciones de reflexión autogestiva. El alumno entonces reasume el conocimiento teórico impartido durante las lecciones y con él cuestiona su propia percepción inicial $\mathrm{y}$ final del caso (MARTÍNEZ Y OROZCO, 2012, p. 51).

Siguiendo esta directriz de razonamiento, los alumnos aprecian entonces el juicio moral como un esquema de conducta evolutiva a nivel moral para la cual la película establece un caso que expone una diferencia significativa con respecto al desarrollo biológico de un ser humano. Las aportaciones de Kohlberg (ZERPA, 2007, p. 116, que entiende a Kohlberg como sistematización de Piaget), uno de los más destacados analistas de la psicología moral y cuyo basamento teórico complementa la información de mis lecciones sobre juicio moral, apuntan, de hecho, hacia un diseño en el que los estadios morales pueden ser comprendidos en el ámbito de la formación académica de los estudiantes muestra, desde una niñez que les resulta fácilmente juzgable hasta una potencial adultez (BARRA, 1987, p. 8) en la que adquirirán principios éticos universales. En ese sentido, la película podría funcionar para algún otro alumno de Bachillerato como un modelo metacognitivo que no sólo le permita la auto-reflexión desde el aprendizaje basado en un estudio de caso, sino que amplíe también su criterio como actor del proceso de aprendizaje, en general, y del razonamiento moral, en particular.

No obstante, he notado que la consideración metacognitiva suele ser poco percibida con sólo ver la película en un aula de clases sin que haya un espacio de diálogo posterior en el que se incentiven interpretaciones diversas o se enriquezcan las evidentes. Por su parte, el conocimiento autogestivo sí deviene una constante y que se mantiene a lo largo del curso de Ética a través de la aplicación continua del juicio moral, según he expresado antes, como herramienta habilitante que facilita al alumno la consecución de las competencias y los contenidos temáticos subsecuentes.

De hecho, considerando su inserción dentro del sistema de Bachillerato en general, no sólo dentro del ámbito de la formación ética, la película de Miguel Littín ofrece también una ventaja que muy pocas películas me han ofrecido y que la hace realmente didáctica en el ámbito de las Humanidades: problematiza (MARTÍNEZ Y OROZCO, 2012, p. 54) sobre la base de un suceso histórico que se somete a interpretación $\mathrm{y}$, en consecuencia, a una revisión que fomenta una diversidad de cuestionamientos morales que a veces caen en el terreno de la sobrein- 
terpretación

(MARTÍNEZ-

SALANOVA, 1998, p. 31); no obstante, esta abundancia de interpretaciones, paradójicamente, tiende más bien hacia a una línea de razonamiento particular que he percibido a través de los escritos que presentaron mis estudiantes muestra: esta película confirma el funcionamiento que tiene a priori el significado del término "moral" (o "la moral", si se quiere), coincidente con el enfoque que postulan Piaget $y$ Kohlberg con respecto al crecimiento y desarrollo intelectual (BONILLA, 2005, p. 63 y 76-77): las nociones del bien y el mal son subjetivas e incluso aparecen relativas en contextos dentro de los que se expone un conflicto en el plano de la realidad.

Por lo tanto, la moral viene a significar la versión que cada individuo tiene sobre lo que está bien o mal; en la película, el personaje Jorge Valenzuela, desde su escaso desarrollo moral, juzgaba las relaciones humanas no con base en esquemas sociales y regulaciones sistémicas, como se espera de un ser humano en el estadio 4, sino a partir de un mero individualismo instrumental (BARRA, 1987, p. 12) y una ingenua perspectiva interpersonal.

\section{El momento de intervención didáctica}

El chacal de Nahueltoro narra la escena del asesinato de la viuda y sus hijos a manos de Jorge Valenzuela de forma simple, con un montaje en el que persiste el subtexto de la ingenuidad moral, aunque por medio de efectos estéticos que acentúan un tono trágico para el espectador, incluso si co- noce los sucesos históricos. Se nos hace saber que Jorge Valenzuela llegó a sentir cariño por la familia a la que asesinó; la mujer, llamada Rosa, le ofreció un vaso de agua y comida mientras el hombre vagaba por el lugar $\mathrm{y}$, luego de que las fuerzas del orden público la desahuciaron, aquél la acompañó en su peregrinar. Mató a los niños, dijo a la prensa, porque estaban solos y no quería que sufrieran más.

Los alumnos encontraron extraña la acción de Jorge Valenzuela cuando éste les puso piedras en las manos a los cadáveres durante aquella escena, por lo que les pregunté, mientras la escena corre (es una escena que no tiene diálogos y permite una intervención del docente sin distraer al alumno), qué pensaban que significase esa acción. Lo que contestaron en su mayoría fue que les parecía un acto bastante ingenuo, incluso torpe, o que el director de la película había querido acentuar la escasez moral del personaje haciendo que efectúe un acto de connotaciones "rituales" en las que se trasluce un intento poco racional por entender a la muerte. De hecho, en un flashforward, mientras la prensa lo interroga al respecto de las piedras en las manos de los cadáveres, Jorge Valenzuela responde que lo hizo porque no quería que se movieran.

Todo este punto de trama fue crucial para interpretar el grado de aprendizaje que han tenido los estudiantes y me permitió saber que el método de estudio de caso, para este tema, favorece el aprendizaje cuando se lleva a cabo durante la fase de desarrollo o de cierre en una secuencia didáctica. Para los fines que perseguía 
con la presente investigación, la película se proyectó a la muestra estudiantil durante la fase de cierre, por lo que noté que un había un conocimiento teórico bastante afianzado y, por ende, el caso que la película resalta presentó mayor facilidad de interpretación que en fases de desarrollo que había ensayado en cursos de Ética precedentes.

Durante esta fase didáctica, la película introdujo lo que BELLIDO expresa sobre el cine en términos de "una representación del mundo, una realidad, unos seres" (1998, p. 15); es decir, la concepción del juicio moral más concreta y contextualizda en una realidad que los alumnos pueden percibir directamente; las interacciones de grupo con las dinámicas de exposición del docente tendieron a una mayor claridad al considerar el caso de la película como un referente modélico al que se le pueden aunar comparaciones o paralelismos con situaciones personales u otros materiales fílmicos.

Un ejemplo que resultó esclarecedor y congruente con este uso de la película en la fase de cierre lo ofrece su desenlace: Jorge Valenzuela es fusilado, cumpliendo su condena por asesinato; sin embargo, antes de que lo fusilaran, el hombre había dedicado su tiempo a estudiar en prisión, haciéndose progresivamente más consciente de su crimen. Podemos afirmar, por ende, que Jorge Valenzuela había evolucionado moralmente al educarse, según lo que se ha establecido con respecto al fundamento teórico de Piaget y Kohlberg. Fue posible, pues, marcar para el alumno momentos en los que cada etapa del juicio moral se encuentre resaltada a través de ejemplos en si- tuaciones cotidianas con miras a obtener de ellos una opinión acerca de la justicia o injusticia del fusilamiento e inducir en ellos una búsqueda de esos patrones de conducta en individuos de las edades correspondientes para que los describieran. Con base en esta metodología, la fase de cierre permite la elaboración de productos de análisis más extensos, como el ensayo o la reseña crítica. Lo que DEL MORAL señala precisamente hablando de las aulas de Ética, aporta también una forma en que se hace funcional trabajar este diseño estableciendo la cinta en el cierre: "La capacidad para ver desde varios lados, a menudo opuestos, una misma historia es una habilidad básica que todo el mundo necesita para desarrollar y afinar sus percepciones de la realidad" (1997, p. 150).

Sin embargo, la fase de cierre provee la entrada a otras dimensiones didácticas. Si consideramos al cine como un material que fomenta la transversalidad con otras asignaturas o con otras áreas curriculares (MARTÍNEZ-SALANOVA, 2003, p. 48), entonces ver El chacal de Nahueltoro durante al cierre del tema es un buen auxiliar (RUÍZ, 1994, p. 75), pues la intención que pretende el filme de mostrarnos un evento trágico dramatizado con tintes de crítica y uso de un profundo discurso ideológico puede conducirse a otras áreas de estudio, como la literatura o la economía, con el fin de juzgar también las implicaciones y efectos que plantea. La escena del desahucio puede ser el detonante de un debate sobre sistemas de gobierno, pobreza o historia; la escena de la interacción en prisión de Jorge Va- 
lenzuela con otros reos puede llevarse al plano de la sociología o de la psicología; las escenas en que los medios de comunicación vierten sus prematuras opiniones al público podrían determinar proyectos interdisciplinares con estadística, comunicación o lógica..

Dentro de este diseño, puesto que la película como espacio también de entretenimiento y captación de interés (MARTÍNEZ-SALANOVA, 1998, p. 28, 31; RUÍZ, 1994, p. 74; DEL MORAL, 1997, p. 149-150; GRUP EMBOLIC, 1998, p. 78), noté que la información sobre el juicio moral fomenta estrategias de interacción lúdica para la apertura y de generación de opiniones y lluvias de ideas para el desarrollo; la utilización de organizadores gráficos, como el diagrama de Venn o los cuadros sinópticos facilitan la compenetración de un conocimiento teórico sobre juicio moral anterior al estudio de caso a través de la película.

Didácticamente, este fomento de la transversalidad e inclusión de entretenimiento implica un mayor acercamiento a modelos educativos de inclusión del alumno en procesos interdisciplinares y "situaciones didácticas relacionadas con otros conocimientos" (MARTÍNEZ-SALANOVA, 2003, p. 47), por lo que justifica a la vez el hecho de ver al cine como una herramienta cognitiva que exige a la vez preparación e información profunda (GÓMEZ, 1996, p. 129-130).

Esta metodología de enseñanza y la aplicación del aprendizaje basado en estudio de caso, pues, me llevan a inferir que un visionado completo y analítico de El chacal de Nahueltoro durante la etapa de cierre resulta en una teorización del tema más asequible para los alumnos que constituyeron la muestra de este estudio.

Es por eso que la relevancia de enseñar el juicio moral usando El chacal de Nahueltoro estriba en la universalidad de su conflicto central y en su adaptabilidad a la esfera de conocimiento planteada por Piaget y Kohlberg, al expresar un dilema mediante una narrativa sencilla, pero llena de significado, de manera análoga a lo que expresa para MARTÍNEZSALANOVA la película italiana $\mathrm{Pa}$ dre padrone (2003, p. 50-51).

\section{Conclusiones}

En un aula de clases para alumnos de Preparatoria, Bachillerato o equivalentes, la enseñanza de la Ética está fundamentada y condicionada por una enseñanza efectiva del tema de juicio moral, por lo que es oportuno encontrar una vía de transmisión de este conocimiento a los estudiantes, de modo que no sólo resulte interesante para ellos, sino también conmovedora y empática. Los resultados de la presente investigación me permitieron interpretar que la mejor forma de enseñar juicio moral es mediante el análisis de una película relevante para cualquier campo de las Humanidades y las Ciencias Sociales, pero que en el particular ámbito de la enseñanza de la Ética adquiere un matiz profundamente esclarecedor sobre la base teórica de los estudios de Jean Piaget y Lawrence Kohlberg.

La película en cuestión, intitulada El chacal de Nahueltoro, plantea un conflicto moral en el que los valo- 
res de excepción a las condiciones humanas esperadas permiten la reflexión y la reconsideración de los sistemas de valores que los alumnos mismos analizaron el film; la evolución moral y psicológica que experimenta el personaje, aunado a la presentación de un discurso de crítica política, informativa y educativa, destacaron una adquisición fructífera de conocimientos y la obtención de una herramienta indispensable en la formación ética de Bachillerato.

Del mismo modo, un diseño estratégico sobre el momento de una planeación en que el film puede ser proyectado en el aula de clases puede adaptarse a las condiciones de un grupo en específico y obedecer a metas didácticas particulares que el profesor requiera. En la aplicación particular de mi diseño estratégico con la población estudiantil que constituyó la muestra del presente estudio, me fue posible verificar la veracidad de mi hipótesis y así comprobar que los objetivos didácticos, como el autoconocimiento o la transversalidad, son asequibles dentro del aprendizaje basado en estudio de caso, resaltando la condición de "imprescindible" para este tipo de contenidos.

Por medio de un análisis detallado de la cinta durante la fase de cierre, los alumnos tuvieron la oportunidad de hacer interpretaciones enriquecedoras, por lo que se fomentó en ellos una clara comprensión sobre el funcionamiento de los estadios del juicio moral y de ña noción misma del término "moral", lo cual se notó en su capacidad para juzgar acciones, contextualizarlas, someterlas a comparación y plantearlas en los subsecuentes temas de la asignatura, como la sexualidad, el relativismo cultural o la bioética. Los alumnos de este nivel de estudios han demostrado también la obtención de un grado mayor de autoconsciencia moral e incluso han sido capaces de arrastrar a otros campos disciplinares el contenido de este tema al considerar el aprendizaje del juicio moral mediante una película como una competencia habilitante y un fundamento universal. 


\section{Referencias:}

BARRA, E. El desarrollo moral: una introducción a la teoría de Kohlberg. Revista Latinoamericana de Psicología. V. 19. N. 1. 1987. Colombia: Fundación Universitaria Konrad Lorenz.

BELLIDO, A. El aprendizaje del cine. Comunicar. N. 11. 1998. España: Grupo Comunicar.

BONILLA, Á. R. Análisis comparativo de cinco teorías sobre el desarrollo moral. Bogotá: Pontificia Universidad Javeriana, 2005.

DEL MORAL, A. Aproximación al análisis de películas en las aulas de Ética. Comunicar. N. 9. 1997. España: Grupo Comunicar.

GRUP EMBOLIC. Cómo enseñar filosofía con la ayuda del cine. Comunicar. N. 11. 1998. España: Grupo Comunicar.

GÓMEZ, J. L. Psicología, cine y educación. Comunicar. N. 7. 1996. España: Grupo Comunicar.

KOHLBERG, L. Psicología del desarrollo moral. Bilbao: Desclée de Brouwer, 1992.

LITTÍN, M. Título Original: El chacal de Nahueltoro; País de Origen: Chile. Estudio/Distrib.: CENTRO DE CINE EXPERIMENTAL DE UNIVERSIDAD DE CHILE, CINEMATOGRÁFICA TERCER MONDO (1969).

MARTÍNEZ, A. y OROZCO, J. H. Cine y educación - Campo de visión, movimiento, velocidad y poder. Revista Colombiana de Educación. N. 63. 2012. Bogotá.

MARTÍNEZ-SALANOVA, E. Aprender pasándolo de película. Comunicar. N. 11. 1998. España: Grupo Comunicar.

MARTÍNEZ-SALANOVA. El valor del cine para aprender y enseñar. Comunicar. N. 20. 2003. España: Grupo Comunicar.

MIRAVAlleS, L. Cómo disfrutar del cine en el aula. Comunicar. N. 11. 1998. España: Grupo Comunicar.

PÉREZ, E. et al. Orígenes Históricos del libro de Jean Piaget sobre "El juicio moral en el niño": sus fuentes filosóficas y científicas. Revista de Historia de la Psicología. V. 17. N. 3-4. 1996. España. 
PIAGET, J. El criterio moral en el niño. (Vidal, N., Trad.). Barcelona: Martínez Roca, 1987.

RUÍZ, F. Cine y enseñanza. Comunicar. N. 3. 1994. España: Grupo Comunicar.

RUÍZ, F. Preliminares para una didáctica del cine: la detección de ideas previas. Comunicar, N. 11. 1998. España: Grupo Comunicar.

SÁNCHEZ, I. El cine, instrumento para el estudio y la enseñanza de la Historia. Comunicar, N. 13. 1999. España: Grupo Comunicar.

TOBÍAS, M. A., DUARTE, M. C. Y KEMCZINSKI, A. Un repositorio digital de contenido fílmico como recurso didáctico. Comunicar, N. 44. 2015. España: Grupo Comunicar.

VILLEGAS, C. Influencia de Piaget en el estudio del desarrollo moral. Revista Latinoamericana de Psicología. V. 30 N. 2. 1998. Colombia: Fundación Universitaria Konrad Lorenz.

ZEPRA, C. E. Tres teorías del desarrollo del juicio moral: Kohlberg, Rest, Lind. Implicaciones para la formación moral. Laurus. V. 13. N. 23. 2007. Venezuela: Universidad Pedagógica Experimental Libertador.

$O(s)$ autor(es) se responsabiliza(m) pelo conteúdo e opiniões expressos no presente artigo, além disso declara(m) que a pesquisa é original.

Recebido em 12/03/2020

Aprovado em 19/06/2020 